\title{
Copying letters to patients: a study of patients' views
}

\author{
AIMS \& METHOD \\ To investigate patients' views on \\ receiving copies of letters sent by \\ their healthcare professionals, 72 \\ patients were asked about their will- \\ ingness to receive a copy of the letter \\ sent to their general practitioner and \\ about preferences for the type of \\ information to be included in such \\ letters. We also asked what concerns, \\ if any, they had about the process.
}

\section{RESULTS}

Three-quarters of the respondents $(n=55)$ said they would like to receive a copy of the letter. Patients accepted the inclusion of information about their illness but were reluctant for data about their family, work and finances to be included.

\begin{abstract}
CLINICAL IMPLICATIONS
Although the majority of the patients we interviewed wished to have the copy letter, many of them expressed concerns about confidentiality, the risk of distress and the cost of the process to the National Health Service. The rights of those who do not want copy letters should also be respected.
\end{abstract}

In the National Health Service (NHS) Plan, the government has made a commitment to send patients copies of letters written between clinicians (Department of Health, 2000). The Good Practice Guidelines issued by the Department of Health (2003) advised that as a general rule (and where patients agreed), letters written by one healthcare professional to another should be copied to the patients concerned. Studies asking the opinions of patients who were already receiving written information (Asch et al, 1991; Slaney \& Vaughan, 1998; Lloyd, 2004; Nandhra et al, 2004) showed that at least $80 \%$ of patients supported the idea. A lower positive response (51-61\%) has been reported in groups who had never received correspondence about their illness (Asch et al, 1991; Thomas, 1998).

We decided to undertake a survey in an out-patient clinic at the Caludon Centre, Coventry, to determine whether patients who had never received a letter from their healthcare professionals would like to receive a copy of the letter sent to their general practitioner, what information they would like included in the letter and what concerns, if any, they had regarding these letters.

\section{Method}

All patients attending a follow-up clinic run by one of the authors (M.M.) in a 4-week period during January and February 2004 were included in the study. After each appointment the patient's written consent was obtained and interviews were completed. These were conducted using a questionnaire designed specifically for the study (further information available from the authors upon request). The questionnaire was tested in a pilot study involving 8 patients and subsequently modified to make it clearer for the respondents.

\section{Results}

During the study period 84 patients had follow-up appointments, out of these 8 (10\%) did not attend, leaving 76 patients eligible for the study. Of these, 72 (95\%) gave their consent for participation. Two patients declined to take part, one could not understand the questions because of language difficulties and one was too unwell to be interviewed. There were 34 (47\%) men and 38 (53\%) women, aged 19-69 years (mean 40.4). Forty-one patients $(57 \%)$ had a diagnosis of affective disorder, 14 patients (19\%) had a diagnosis of schizophrenia/delusional disorder, 10 patients (14\%) had anxiety disorder, 4 patients (6\%) had alcohol-related and other organic disorders and 3 patients (4\%) had personality disorder. They had been in contact with psychiatric services from periods ranging from 1 month to 34 years (mean 5 years 2 months). The average number of outpatient visits made by each patient in the past year was 4.7.

About three-quarters of the patients $(n=55)$ declared that they would like to receive a copy of the letter sent to their general practitioner if it was made available; 15 patients (21\%) did not want to receive a copy of the letter and 2 (3\%) respondents were unsure. We did not find any correlation between the patients' diagnosis and their preference for receiving copy letters. Respondents had different preferences as to the kind of information they would like to be included or excluded from their copy of the letter (Table 1). The majority accepted the inclusion of clinical information in the letters; however, they were more reluctant to include information about their family, employment and financial situations. In the group who would like to receive copy letters, 43 patients had no concern related to the letters, but 12 patients expressed some conditions under which the letters should be sent. Five were worried about confidentiality issues, 2 did not want their general practitioner to see the letter at all and 2 wanted to review letters personally before they were sent. The others had concerns about possible distress caused by the content of the letters, that they would not be able to understand medical terminology in the letter and that sending letters would be expensive for the NHS.

In the group of 15 patients who did not want copy letters, 6 patients thought the letters represented 
unnecessary paperwork and bureaucracy for the NHS and 6 patients believed that their good relationship with their doctor and the amount of information they already received made the letter unnecessary. There were also those who felt the risk of possible distress and breaches of confidentiality ( 5 and 3 patients respectively) outweighed any potential benefits of getting more information about their illness.

\section{Discussion}

Despite the variety of survey methods used in different studies, all results show a consistently high level of support for the provision of written information to patients. In our study $76 \%$ of the respondents wanted copies of the letter sent to their general practitioner after an out-patient consultation. Just under a quarter said they did not want a copy. Our positive response rate is similar to those of Nandhra et al (2004), Lloyd (2004), Slaney \& Vaughan (1998) and Asch et al (1991), despite our patients having no previous experience of receiving written information. It is also slightly higher than the response reported by Thomas (1998) for a similar study group but unlike Thomas's study, diagnosis did not appear to be significant in the decision to receive copy letters in our sample.

Nearly three-quarters of our sample (53/72; 74\%) could not accurately remember the number of outpatient appointments they had attended in the past year and more than half $(39 / 72 ; 54 \%)$ were not aware that a letter was sent to their general practitioner after each appointment. Copying letters to patients might help them to become better informed about their treatment, but whether it would improve treatment outcomes remains unclear. Asch et al (1991) noted that receiving letters did not improve compliance with therapeutic advice, and Bernadt et al (1991) reported that only 3 of their patients spontaneously discussed written summaries with their general practitioner.

Preferences for the type of information in the letter showed a general agreement concerning the inclusion of information about diagnosis, prognosis, medications and mental and physical health problems. There was less acceptance of information about family, finances and work or employment. Lloyd (2004) found that patients

\section{Table 1. Patients' preferences for information type}

\begin{tabular}{|c|c|c|c|}
\hline Information & Include & Exclude & Unsure \\
\hline Diagnosis & 54 & 1 & \\
\hline Medication & 52 & 3 & \\
\hline Prognosis & 51 & 2 & 2 \\
\hline History of psychiatric problems & 50 & 5 & \\
\hline Physical health problems & 50 & 4 & 1 \\
\hline Family situation & 44 & 10 & 1 \\
\hline Work/employment ${ }^{1}$ & 33 & 9 & 1 \\
\hline Financial & 30 & 22 & 3 \\
\hline
\end{tabular}

1. Excluding 12 patients who described themselves as retired or unlikely ever to work. were generally happy with the information in the letters that they received but that they did not want more information about family history. Information about family may prove to be a particularly sensitive area for some patients.

Approximately $10 \%$ of our sample, most of whom did not wish to receive copy letters, had a concern about the impact of the initiative on the health service, a finding not reported elsewhere. Worries about confidentiality and fear of distress from the information contained in letters were reported by our patients; Asch et al (1991) reported similar concerns about disclosures of information in their study. Patients wanted to be protected from a breach of confidentiality, and NHS trusts would need accurate record-keeping systems to ensure such events do not occur. The minimisation of possible distress caused by copy letters has been a concern for patients and psychiatrists alike. Bernadt et al (1991) reported that $28 \%$ of their patients were upset by the content of their letters and Nandhra et al (2004) reported the content of $22 \%$ of the letters sent in their study had to be altered to avoid causing possible distress to the patient. Paradoxically, positive experiences of the service, such as satisfaction with existing information and a good relationship with their doctor, were given as reasons to choose not to receive copy letters in our study.

Copying letters may also generate unexpected demands. Two patients in our sample asked to review their letters personally before they were sent out. Two patients objected to their general practitioner receiving letters about their appointments at all, an objection that has also been reported by Thomas (1998). Furthermore, Dale et al (2003) reported that $75 \%$ of their sample wanted a personalised letter rather than a copy of the letter sent to their general practitioner. It seems that more work needs to be done to explain to patients how the new systems would work to avoid potentially damaging misunderstandings and disappointments.

Our study supports the idea that copying letters would be a popular initiative with psychiatric outpatients. Their concerns suggest a need for clearly understandable information in the letters, with due consideration shown for preferences about content, the need for confidentiality and the patients' mental health. The wishes of the minority who did not want to receive copy letters should also be respected.

\section{Declaration of interest}

None.

\section{References}

ASCH, R., PRICE, J. \& HAWKS, G. (1991) Psychiatric outpatients' reactions to summary letters of their consultations. British Journal of Medical Psychology, 64, 3-9.

BERNADT, M., GUNNING, L. \& QUENSTEDT, M. (1991) Patients'access to their own psychiatric records. BMJ, 303, 967

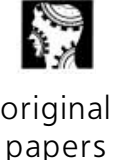

papers 
Practice Guidelines. London: Department of Health (www.doh.gov.uk/patientletters/ original issues.htm).

papers

LLOYD, G. (2004) Medical records: copying letters to patients. Psychiatric Bulletin, 28, 57-59.
NANDHRA, H. S., MURRAY, G. K., HYMAS, N. J., et al (2004) Medical records: doctor and patients' experiences of copying letters to patients. Psychiatric Bulletin, 28 40-42.
SLANEY, M. \& VAUGHAN, P. (1998) THOMAS, P. (1998) Writing letters to Patient access to psychiatric assessment patients. Psychiatric Bulletin, 22 reports. Psychiatric Care, 5, 225-227. 542-545.

* Marek Marzanski Consultant Psychiatrist, Coventry Teaching Primary Care Trust, Caludon Centre, Clifford Bridge Road, Coventry CV2 2TE, Padmapriya Musunuri Senior House Officer, CoventryTeaching Primary CareTrust, Tim Coupe Clinical Effectiveness Officer, Coventry Teaching Primary Care Trust 